University of Nebraska - Lincoln

DigitalCommons@University of Nebraska - Lincoln

Faculty Publications, Department of Psychology

Psychology, Department of

2020

\title{
Maternal Depression and Breastfeeding in Home Visitation
}

Francesca A. Scheiber

University of lowa, francesca-scheiber@uiowa.edu

Lisa S. Segre

University of lowa, lisa-segre@uiowa.edu

Michael W. O'Hara

University of lowa, mike-ohara@uiowa.edu

Darby Taylor

EveryStep, Des Moines, lowa, dtaylor@everystep.org

Rebecca L. Brock

University of Nebraska-Lincoln, rebecca.brock@unl.edu

Follow this and additional works at: https://digitalcommons.unl.edu/psychfacpub

Part of the Psychology Commons

Scheiber, Francesca A.; Segre, Lisa S.; O'Hara, Michael W.; Taylor, Darby; and Brock, Rebecca L., "Maternal Depression and Breastfeeding in Home Visitation" (2020). Faculty Publications, Department of Psychology. 1050.

https://digitalcommons.unl.edu/psychfacpub/1050

This Article is brought to you for free and open access by the Psychology, Department of at DigitalCommons@University of Nebraska - Lincoln. It has been accepted for inclusion in Faculty Publications, Department of Psychology by an authorized administrator of DigitalCommons@University of Nebraska - Lincoln. 


\title{
Maternal Depression and Breastfeeding in Home Visitation
}

\author{
Francesca A. Scheiber, ${ }^{1}$ Lisa S. Segre,${ }^{1}$ Michael W. O'Hara, ${ }^{2}$ Darby Taylor, ${ }^{3}$ \\ and Rebecca L. Brock ${ }^{4}$
}

1. University of Iowa, College of Nursing, Iowa City, Iowa, USA

2. University of Iowa, Department of Psychological and Brain Sciences, Iowa City, Iowa, USA

3. EveryStep, Des Moines, Iowa, USA

4. University of Nebraska-Lincoln, Department of Psychology, Lincoln, Nebraska, USA

Corresponding author - Francesca A. Scheiber, email francesca-scheiber@uiowa.edu

Also: L. S. Segre, email lisa-segre@uiowa.edu, M. W. O’Hara, email mike-ohara@uiowa.edu, D. Taylor, email dtaylor@everystep.org, and R. L. Brock, email rebecca.brock@unl.edu

\begin{abstract}
Background: Home visitation is a popular mechanism for supporting parents and their young children. Breastfeeding is often promoted by home visitors due to its health benefits. However, maternal depression may interfere with breastfeeding. Thus, home visitors may be attempting to encourage health-promoting behaviors like breastfeeding, but maternal depression may interfere with engagement in those behaviors. Method: The data for this study were provided by the Des Moines Healthy Start and the Empowerment Family Support Project (DMHSP). We analyzed the relation between depression and breastfeeding for 364 women. Results: First, rates of elevated depression scores in this sample of women $(8.7 \%-21.4 \%$ of women) were lower than rates of elevated depression scores reported in other studies of women enrolled in home visitation (30\%-50\% of women). Second, rates of breastfeeding at 3 months postpartum in this sample (56\% of women) were higher than rates of breastfeeding reported in the general population (51\% of women). Third, Non-Hispanic White women were significantly less likely to breastfeed compared to other racial groups. Fourth, average Edinburgh Postnatal Depression Scale (EPDS) scores during the first 3 months postpartum, but not during pregnancy, were predictive of likelihood to breastfeed at 3 months postpartum, even after
\end{abstract}


accounting for demographic characteristics. Conclusion: Addressing maternal depression during the early postpartum period may increase the likelihood of engaging in breastfeeding.

Keywords: breastfeeding, home visitation, postpartum depression, maternal depression, parenting practices

\section{Introduction}

Home visitation (HV) is an increasingly popular mechanism for supporting parents and their young children. In 2017, over 300,000 families received HV services via 15 different evidence-based models (National Home Visiting Resource Center, 2018). Although there are various models of $\mathrm{HV}$ programs, most programs inherently share a focus on in-home services aimed at promoting children's health, bolstering children's emotional and academic development, and encouraging family self-sufficiency (Duffee, Mendelsohn, Kuo, Legano, \& Earls, 2017). Most programs target families that are faced with risk factors known to interfere with children's success, such as low socioeconomic status and inadequate prenatal care. Research has shown that HV is effective for a number of outcomes, including promoting positive parent-child interactions, promoting positive parenting behaviors, increasing the likelihood of appropriate socioemotional and cognitive development, and reducing children's healthcare encounters (Kitzman et al., 1997; Love et al., 2005; Mejdoubi et al., 2014; Olds et al., 2002).

Among several parenting practices, increasing the likelihood of breastfeeding has been a target for many HV programs due to its health benefits (Victora et al., 2016). For children, breastfeeding has been shown to have cognitive benefits, offer protection from infections, and reduce the likelihood of obesity and diabetes (Binns, Lee, \& Low, 2016; Victora et al., 2016). For mothers, breastfeeding has been shown to reduce the likelihood of hypertension, diabetes, and cancers (Binns et al., 2016; Stuebe, Rich-Edwards, Willett, Manson, \& Michels, 2005). For families, breastfeeding has been shown to increase spacing between children (Victora et al., 2016).

Due to these health benefits, increasing the rate of breastfeeding is both a national and a global priority. The World Health Organization (WHO) and the American Academy of Pediatrics (AAP), for example, recommend exclusive breastfeeding for the first 6 months of life (American Academy of Pediatrics Section on Breastfeeding, 2012; World Health Organization, 2011). One of the Healthy People 2020 goals is to have $25.5 \%$ of children exclusively breastfed through 6 months postpartum and to have $60.6 \%$ of children receive at least some breastfeeding through 6 months postpartum. In 2015, those rates were, on average, $24.9 \%$ and $57.6 \%$, respectively. Those with the lowest family income and lowest education, similar to those typically enrolled in HV programs, were the least likely to both breastfeed exclusively and breastfeed at all at 6 months postpartum. In 2015, 18.9\% of those with a high school degree, compared to $34.4 \%$ of those with an advanced degree, were exclusively breastfeeding at 6 months postpartum; and $45 \%$ of those with a high school degree, compared to $77 \%$ of those with an advanced degree, were breastfeeding at all at 6 months postpartum (Healthy People, 2020). 
Home visitors are well-positioned to contribute to these goals and priorities due to their frequent contact with mothers both during the prenatal period and the postpartum period. Indeed, HV programs have already been shown to increase the likelihood of initiating breastfeeding (Shah \& Austin, 2014). Given the potential impact of increasing the likelihood of breastfeeding, it is important to understand not only the factors and circumstances that contribute to the likelihood of breastfeeding but also those that undermine that likelihood. One factor that could undermine the likelihood of engaging with breastfeeding, especially in a HV population, is maternal depression (MD).

In a study on the prevalence of MD in HV, Stevens, Ammerman, Putnam, and Van Ginkel (2002) found that around 30\% of women receiving HV services had elevated scores on the Beck Depression Inventory II, a measure of depressive symptoms. Similarly, Segre, O'Hara, Brock, and Taylor (2012) found that around 30\% of women receiving HV services had elevated scores on the Edinburgh Postnatal Depression Scale (EPDS), a measure of depressive symptoms used during the perinatal period. In a review of MD in HV, Ammerman, Putnam, Bosse, Teeters, and Van Ginkel (2010) reported a study in which up to a 50\% of women enrolled in HV experienced elevated levels of depressive symptoms. These rates are much higher than those typically found in the perinatal population, which hovers between 13\% (O'Hara \& Swain, 1996) and 19\% (Gavin et al., 2005). It is important to investigate depressive symptoms in the context of HV because HV attempts to engage mothers in parenting practices, including breastfeeding, that require the exact tools-energy and motivation - that depressive symptoms may compromise.

Maternal depression has previously been cited as one factor that interferes with breastfeeding (Hatton et al., 2005; Slomian, Honvo, Emonts, Reginster, \& Bruyere, 2019), specifically its initiation and duration (Dennis \& McQueen, 2009; Dias \& Figueiredo, 2015). Studies have also demonstrated a relation between MD and attitudes about breastfeeding, feelings of competence, and difficulties breastfeeding (Galler, Harrison, Ramsey, Chawla, \& Taylor, 2006; Henderson, Evans, Straton, Priest, \& Hagan, 2003; Watkins, Meltzer-Brody, Zolnoun, \& Stuebe, 2011).

Taken together, prior research suggests that while home visitors may be attempting to encourage breastfeeding, MD may interfere with the likelihood of breastfeeding. While previous research has demonstrated elevated levels of MD in HV studies, there is a paucity of research focused on the potential undermining effects of MD on breastfeeding in HV. The purpose of the present study was to examine whether MD might undermine the likelihood of breastfeeding, specifically in a HV population. Results from this study have the potential to inform future prevention and intervention efforts in HV related to breastfeeding practices in women who experience depressive symptoms.

\section{Method}

In the present study, we ask whether rates of MD are similar to rates of depression reported in other HV programs, how rates of breastfeeding compare to rates of breastfeeding reported nationally, and whether severity of MD predicts the likelihood of breastfeeding. 


\subsection{Setting}

The data for the present study were provided by the Des Moines Healthy Start and the Empowerment Family Support Project (DMHSP), which operates through EveryStep. This program provides HV services for women located in geographic areas with high infant mortality rates. Most women in DMHSP face risk factors including a low socioeconomic status and inadequate healthcare. Parents may enroll in the program prenatally or postnatally; parents do not have to be first-time parents to enroll. Once enrolled, parents are able to receive home visits until their child is 5 years old. As part of DMHSP, home visitors use evidence-based curricula-Growing Great Kids and Parents as Teachers-to educate parents, and sometimes other caregivers, about caregiving practices that optimize child development and well-being. Regarding breastfeeding, home visitors provide caregivers with education on several feeding options, including on the benefits and consequences of each type of feeding option. Although guided by the curricula, home visitors tailor sessions to families' specific needs and provide additional advocacy and support for families if requested.

DMHSP also provides prenatal and postnatal appointments with a nurse consultant during the third trimester and during the first two weeks postpartum. Among several topics related to pregnancy, child birth, and child development, the nurse consultant provides education on breastfeeding. The nurse consultant also assists participants struggling with breastfeeding by providing one-on-one appointments to resolve breastfeeding challenges.

Administrators from DMHSP provided demographic, depression, parenting, and child outcome data from 2,480 women who enrolled between 2001 and 2010. Home visitors collected demographic information upon enrollment. They aimed to collect information on MD at several assessment periods: 1st, 2nd, and 3rd trimesters of pregnancy, as well as 3, $6,9,12,18$, and 24 months postpartum. They also aimed to collect parenting and child information at the same postpartum assessment periods: 3, 6, 9, 12, 18, and 24 months postpartum. However, because participants could enroll in DMHSP at various times throughout pregnancy and the postpartum period, the number of assessments for each participant was highly variable.

\subsection{Participants}

The database provided by DMHSP included information from a total of 2,480 women. Not all women in the database provided data that aligned with the aims of this paper. As such, we selected women who provided data on breastfeeding at 3 months postpartum, at least one depression score during pregnancy, and at least one depression score during the first 3 months postpartum. Using these criteria, we selected 364 women for our final sample.

Compared to other women in the database $(N=2106)$, the women in our sample $(N=364)$ were less likely to be Non-Hispanic White, $\chi^{2}(1)=10.988, p=.001$, more likely to be in a relationship, $\chi^{2}(1)=20.841, p<.001$, less likely to be insured, $\chi^{2}(1)=8.850, p=.003$, and of older age, $t(558.83)=-4.076, p<.001$. Importantly, the groups did not significantly differ on rates of breastfeeding at 3 months postpartum, $\chi^{2}(1)=1.116, p=.291$. There were also nonsignificant differences in severity of depression during pregnancy, $t(518)=1.177, p=$ .240; however, women who were not selected for this study had higher depression scores 
during the first 3 months postpartum, $t(848.47)=2.753, p=.006$. The demographic characteristics of the mothers in the present study are displayed in Table 1.

\begin{tabular}{|c|c|c|}
\hline & & $\mathrm{M}(\mathrm{SD})$ \\
\hline \multirow[t]{2}{*}{ Maternal Age at Birth } & & $27.55(5.71)$ \\
\hline & & $N(\%)$ \\
\hline Child Gender & Female & $187(51.4 \%)$ \\
\hline \multirow[t]{6}{*}{ Race } & Non-Hispanic Asian & $65(17.9 \%)$ \\
\hline & Non-Hispanic Black & $81(22.3 \%)$ \\
\hline & Non-Hispanic White & $51(14.0 \%)$ \\
\hline & Non-Hispanic Other & $7(1.9 \%)$ \\
\hline & Hispanic White & $142(39.0 \%)$ \\
\hline & Hispanic Other & $18(4.9 \%)$ \\
\hline In a Relationship & Yes & $246(67.6 \%)$ \\
\hline Insured & Yes & $174(48.3 \%)$ \\
\hline High School Education & Yes & $149(40.9 \%)$ \\
\hline
\end{tabular}

\subsection{Variables}

In the present study, there was one dependent variable-breastfeeding at 3 months postpartum - and one independent variable-maternal depression - per analysis.

\subsubsection{Depression symptom severity}

The Edinburgh Postnatal Depression Scale (EPDS) (Cox, Holden, \& Sagovsky, 1987) was used by DMHSP home visitors to screen for depression. The EPDS is a 10-item self-report measure that assesses the severity of depressive symptoms. Participants responded to each item on a scale of 0 to 3 . Scores range from 0 to 30, with higher scores reflecting higher depressive symptomology. The EPDS has been validated in a perinatal population and has adequate sensitivity (86\%) and specificity (78\%) (Cox et al., 1987). The EPDS is widely used and has high internal consistency (Bergink et al., 2011; McBride, Wiens, McDonald, Cox, \& Chan, 2014).

DMHSP has previously used a cutoff score of $\geq 12$ on the EPDS to indicate likely depression (Segre et al., 2012); we used the same cutoff score in the present study. We computed the severity of depressive symptoms by averaging mothers' available EPDS scores during the prenatal period and separately during the early postpartum period. Out of three total possible EPDS scores during pregnancy (1st trimester, 2nd trimester, and 3rd trimester), women had an average of $1.3(S D=0.5$, range $=1-2)$ scores. Out of three total possible EPDS scores during the first 3 months postpartum (1-3 weeks postpartum, 6 weeks postpartum, 3 months postpartum), women had an average of 1.9 ( $S D=0.4$, range =1-3) scores. It should be noted that, for some women, their average score reflected one score. However, EPDS scores at different time points were correlated with each other (Table 2). 
Table 2. Bivariate Correlations between EPDS Scores at Different Assessment Periods

\begin{tabular}{llcccc}
\hline & 1st Tri & 2nd Tri & $1-3 \mathrm{~W} \mathrm{PP}$ & $6 \mathrm{~W}$ PP & $3 \mathrm{M} \mathrm{PP}$ \\
\hline \multirow{2}{*}{ 1st Tri } & Pearson Correlation & $0.625^{* *}$ & $0.477^{* *}$ & 0.062 & $0.371^{* *}$ \\
& $N$ & 103 & 101 & 3 & 99 \\
\multirow{2}{*}{ 2nd Tri } & Pearson Correlation & & $0.506^{* *}$ & 0.399 & $0.447^{* *}$ \\
& $N$ & 337 & 14 & 330 \\
$1-3 \mathrm{~W}$ PP & Pearson Correlation & & & $0.830^{*}$ & $0.596^{* *}$ \\
& $N$ & & 12 & 308 \\
6 W PP & Pearson Correlation & & & $0.674^{*}$ \\
& $N$ & & & 13
\end{tabular}

${ }^{* *} p<.001,{ }^{*} p<.05 ;$ Tri $=$ trimester, $\mathrm{PP}=$ postpartum, $\mathrm{W}=$ weeks, $\mathrm{M}=$ months

\subsubsection{Breastfeeding}

During the postpartum period, home visitors asked mothers, "Are you currently breastfeeding?" Mothers could respond "yes," "no," or "never breastfed." We dichotomized this variable into "yes" (scored 1) and "no" (scored 0) by grouping together "never breastfed" with answers of "no."

For the present analysis, we used data reported at the 3 month postpartum assessment. We neither had information on mothers' employment status at each assessment period nor information on whether mothers were offered a paid maternity leave, thus we chose the 3 month postpartum assessment period because we believe it is the assessment period during which mothers were most likely to still be at home with their children, due to a typical maternity leave in the United States lasting 3 months. Returning to work has been shown to decrease the likelihood of breastfeeding (Laisiriruangrai, Wiriyasirivaj, Phaloprakarn, \& Manusirivithaya, 2008; Ogbuanu, Glover, Probst, Liu, \& Hussey, 2011), thus examining the 3 month assessment period instead of the 6 month assessment period, for example, potentially reduced some confounds associated with returning to work. Moreover, even though the $\mathrm{WHO}$ and the AAP recommend exclusive breastfeeding through 6 months postpartum, breastfeeding for at least 3 months postpartum is also predictive of several important health outcomes (Chung et al., 2007).

\subsubsection{Covariates}

Various demographic variables (race, relationship status, insurance, and maternal age at birth) were significantly associated with breastfeeding at 3 months postpartum (Table 3); thus, we included them as covariates in each analysis. A chi-square test indicated the relation between race and breastfeeding at 3 months postpartum was statistically significant, $\chi^{2}(5),(N=364)=19.742, p=.001$, and post-hoc analyses revealed that the Non-Hispanic White group was driving this finding (Table 4). Due to this result, we dichotomized race as NonHispanic White vs. Other for subsequent analyses. Both relationship status and insurance status were dichotomized (being in a relationship vs. not; having insurance vs. not). Maternal age at birth was significantly associated with breastfeeding at 3 months postpartum; mothers who breastfed had a mean age of 28.14 years, and mothers who did not breastfeed had a mean age of 26.79 years, $t(362)=-2.26, p=.024$. 
Table 3. Relationship between Demographic Characteristics and Breastfeeding

\begin{tabular}{lccc}
\hline Demographic Characteristic & BF Yes/No & Percentage & Group Comparisons \\
\hline Non-Hispanic White & yes & $27.5 \%$ & $\chi^{2}(1, N=364)=19.683, p<.001$ \\
& no & $60.7 \%$ & \\
In a relationship & yes & $61.4 \%$ & $\chi^{2}(1, N=364)=8.778, p=.003$ \\
& no & $44.9 \%$ & \\
Insurance & yes & $48.9 \%$ & $\chi^{2}(1, N=360)=6.132, p=.013$ \\
& no & $61.8 \%$ & \\
High school education & yes & $51.0 \%$ & $\chi^{2}(1, N=364)=2.598, p=.107$ \\
& no & $59.5 \%$ & \\
\hline
\end{tabular}

Note: $\mathrm{BF}=$ breastfeeding

Table 4. Percentage of Each Racial Group Breastfeeding at 3 Months Postpartum

\begin{tabular}{lc}
\hline Race & Percentage \\
\hline Non-Hispanic Asian & $61.5 \%$ \\
Non-Hispanic Black & $60.5 \%$ \\
Hispanic White & $60.6 \%$ \\
Non-Hispanic White & $27.5 \%$ \\
Hispanic Other & $61.1 \%$ \\
Non-Hispanic Other & $57.1 \%$ \\
\hline
\end{tabular}

\section{Results}

\subsection{Results question 1}

What are the descriptive characteristics of depression scores in this sample of women receiving HV services? During pregnancy, the average EPDS score was $6.7(S D=4.86)$, and during the first 3 months postpartum the average EPDS score was $5.4(S D=4.18)$. Table 5 lists further descriptive characteristics of depression scores in this sample of women.

\begin{tabular}{lccccc}
\hline \multicolumn{7}{l}{ Table 5. Descriptive Characteristics of Depression Scores } \\
\hline \multicolumn{1}{l}{ 1st Tri } & 2nd Tri & 1-3 W PP & 6 W PP & 3 M PP \\
\hline Minimum & 0 & 0 & 0 & 0 & 0 \\
Maximum & 23 & 24 & 21 & 15 & 21 \\
Mean (SD) & 6.13 & 6.61 & $5.49(4.72)$ & 6.29 & 5.39 \\
& $(5.12)$ & $(5.00)$ & & $(5.18)$ & $(4.49)$ \\
Percentage of women with EPDS $\geq 12$ & $16 \%$ & $14 \%$ & $11.5 \%$ & $21.4 \%$ & $8.7 \%$ \\
$N$ & 106 & 361 & 338 & 14 & 333 \\
\hline
\end{tabular}

Note: Data for third trimester was not available. Tri $=$ trimester, $\mathrm{PP}=$ postpartum, $\mathrm{W}=$ weeks, $\mathrm{M}=$ months

\subsection{Results question 2}

What are the descriptive characteristics of breastfeeding in this population at 3 months postpartum? At 3 months postpartum, $56 \%$ of mothers in this sample were breastfeeding. As seen in Table 2, NonHispanic White mothers, mothers not in a relationship, mothers 
with insurance, and younger mothers were significantly less likely to breastfeed than their respective counterparts.

\subsection{Results question 3}

Is the severity of maternal depression associated with the likelihood of breastfeeding in this population? A binary logistic regression-with breastfeeding at 3 months as the dependent variable-indicated that, after accounting for race, relationship status, insurance, and maternal age, the severity of depression during pregnancy did not significantly predict breastfeeding at 3 months postpartum, $\chi^{2}(1)=2.606, p=.106$. In this analysis, both race, $\chi^{2}(1)=10.887, p=.001$, and relationship status, $\chi^{2}(1)=4.906, p=.027$, emerged as unique predictors of breastfeeding at 3 months postpartum.

In contrast to the severity of depression during pregnancy, after accounting for race, relationship status, insurance, and maternal age, the severity of depression during the first 3 months postpartum significantly predicted breastfeeding at 3 months postpartum, $\chi^{2}(1)$ $=7.154, p=.007$. In this analysis, both race, $\chi^{2}(1)=12.201, p<.001$, and relationship status, $\chi^{2}(1)=5.500, p=.019$, also emerged as unique predictors of breastfeeding at 3 months postpartum. The severity of depression during the first 3 months postpartum remained a significant predictor of breastfeeding at 3 months postpartum, even when accounting for race, relationship status, insurance, maternal age, and the severity of depression during pregnancy, $\chi^{2}(1)=4.735, p=.030$.

\section{Discussion}

Taken together, this study contextualized rates of depression, rates of breastfeeding, and the relation between depression and breastfeeding in a sample of low-income women enrolled in a HV program. The study produced several noteworthy findings. First, the rates of elevated depression scores in this sample of women $(8.7 \%-21.4 \%)$ were more consistent with rates of depression in the general perinatal population (13\%-19\%) (Gavin et al., 2005; O'Hara \& Swain, 1996) than they were with rates reported in other studies of women enrolled in HV (30\%-50\%) (Ammerman et al., 2010; Segre et al., 2012; Stevens et al., 2002). There are several possible explanations for this finding. As mentioned earlier, mothers who were selected for the present study had lower average EPDS scores during the first 3 months postpartum than mothers (with available data) in the broader dataset (5.38 vs. 6.18; $t(848.47)=2.753, p=.006)$. Moreover, during the years that data for this study were gathered, DMHSP had newly implemented procedures for MD, for which home visitors received training on screening and referral processes. During this time, $64 \%$ of women accepted a referral for treatment, and $47 \%$ of women reported having received treatment; treatment included medication, counseling, and a combination of medication and counseling. (Segre et al., 2012; Segre \& Taylor, 2014). In 2005, DMHSP also implemented Listening Visits, a MD treatment developed for delivery by HV nurses in the UK (Holden, Sagovsky, \& Cox, 1989). DMHSP reported positive evaluation results for Listening Visits in an open and randomized controlled trial (Segre, Brock, \& O'Hara, 2015; Segre, Stasik, O'Hara, \& Arndt, 2010). Thus, about half of mothers with elevated EPDS scores were being connected with relevant mental health resources. 
Second, at 3 months postpartum, rates of breastfeeding in this sample of women (56\%) were higher than the national average $(51 \%)$ during the time period at which these data were collected, especially the national average for women with similar socioeconomic backgrounds (40-48\%) (Li, Darling, Maurice, Barker, \& Grummer-Strawn, 2005). It is possible that women who enroll in HV programs are more likely to breastfeed. However, it is also possible that the breastfeeding education provided by DMHSP home visitors and nurse consultants was successful in increasing the likelihood of breastfeeding for some women. Home visitors in DMHSP provided families with a comprehensive education on the benefits and consequences of various feeding options, including exclusive breastfeeding, partial breastfeeding, or exclusive formula use. Moreover, nurse consultants were available to help participants problem solve difficulties breastfeeding. Research has shown that women with more support, both lay support and professional support, are more likely to breastfeed, more likely to breastfeed for longer, and less likely to discontinue exclusive breastfeeding (Britton et al., 2007; Raj \& Plichta, 1998); therefore, it is possible that home visitors and nurse consultants acted as the support necessary for some women to engage in breastfeeding.

Third, higher EPDS scores during the first 3 months postpartum predicted a lower likelihood of breastfeeding at 3 months postpartum, even after accounting for other demographic characteristics. This suggests that EPDS scores during the postpartum period, when compared to EPDS scores during the prenatal period, may have greater predictive utility in terms of understanding breastfeeding practices. Screening and connecting mothers with mental health resources during this potentially sensitive time may be particularly important. Home visitors in DMHSP promote several other practices, like maintaining upto-date immunization and well-baby visit schedules. It is possible that MD interferes with the likelihood of engaging in these other practices as well.

As mentioned earlier, home visitors were quite successful in screening and referring women whose EPDS scores were $\geq 12$ during this time period; however, about half of the women who had elevated EPDS scores either declined treatment or did not follow up after being referred for treatment (Segre et al., 2012). Due to the finding that MD might undermine the likelihood of breastfeeding, interventions aimed at bridging the gap between referrals and treatment may be particularly productive.

Finally, Non-Hispanic White women were significantly less likely to breastfeed than other racial groups, a finding contrary to results from previous studies in several ways. Previous studies, including those focused on low-income populations, have demonstrated that Hispanic women are more likely to breastfeed than other racial/ethnic groups (Li et al., 2005). Moreover, although some studies suggest that Black women are less likely to breastfeed than White women (Forste, Weiss, \& Lippincott, 2001; Li et al., 2005), other studies suggest no differences in breastfeeding between Black and White women (Hurley, Black, Papas, \& Quigg, 2008). Our sample of women deviated from each of these previous findings; in our sample, Hispanic women were equally as likely to breastfeed as other racial groups (except for White women), Black women were not less likely to breastfeed than other racial groups, and White women were significantly less likely to breastfeed than all other racial groups. Perhaps other relevant characteristics were associated with White women in our sample, making them less likely to breastfeed. However, exploratory 
analyses revealed no significant differences between Non-Hispanic White and Other women regarding characteristics previously shown to be associated with breastfeeding: relationship status, $\chi^{2}(1)=1.251, p=.263$, average depression scores during pregnancy, $t(362)=-0.444, p=.657$, and average depression scores in the first 3 months postpartum, $t(362)=1.02, p=.310$.

\subsection{Limitations}

This study has limitations. The data provided by DMHSP were collected for administrative, rather than research, purposes. Thus, we were limited to using the data that were available in this database to pursue the study aims. There are several other important aspects of breastfeeding that were not included in this study. For example, both the WHO and the AAP distinguish between exclusive breastfeeding and concurrent use of formula; we were not able to make this distinction in our study. Other important aspects of breastfeeding include previous experience breastfeeding (Huang, Ouyang, \& Redding, 2019), attitudes toward breastfeeding (Galler et al., 2006), difficulties breastfeeding (Gianni et al., 2019), feelings of competence regarding breastfeeding (Lau, Lok, \& Tarrant, 2018), and whether mothers received maternity leave (Ogbuanu et al., 2011).

Additionally, the generalizability of our findings is limited by several factors. The DMHSP program exclusively serves impoverished families, thereby limiting the generalizability of our findings to groups of women from other socioeconomic backgrounds. However, this sample represents a racially and ethnically diverse population of women, and our findings can inform research regarding the role of MD in other HV programs with similar populations. Additionally, although DMHSP home visitors incorporate curricula (e.g., Parents as Teachers) that other HV programs also incorporate, DMHSP home visitors tailor visits to families' unique needs, thus findings cannot be generalized to all HV models. Moreover, the sample we selected was less likely to be NonHispanic White, more likely to be in a relationship, and less depressed during the first 3 months postpartum than the sample that was part of the larger database, also limiting the generalizability of findings. Finally, the women who were part of the larger database but were not selected for the final sample due to insufficient data might reflect a group for whom engagement with $\mathrm{HV}$ is more difficult, further limiting generalizability of the results.

\subsection{Future directions}

The current study highlighted several opportunities for future directions. First, future studies should account for the other important aspects of breastfeeding mentioned above in an effort to make more specific recommendations for prevention and intervention efforts aimed at optimizing breastfeeding practices. Second, previous research has demonstrated that treatment of maternal depression alone may not be sufficient to improve parenting (Forman et al., 2007; Goodman, Prager, Goldstein, \& Freeman, 2015; Logsdon, Wisner, \& Hanusa, 2009); however, there have been promising results from interventions that have targeted parenting in the context of maternal depression (Cicchetti, Rogosch, \& Toth, 2000). Since HV is a parenting intervention, productive next steps for future studies might include: (1) examining whether those who receive mental health treatment, and have associated reductions in depressive symptoms, are more likely to breastfeed than those who do 
not receive treatment, and (2) examining whether the timing of depressive symptoms, the timing of treatment, and the timing of breastfeeding education interact with each other to influence the likelihood of breastfeeding. Examining such questions could inform updates to HV programs' existing depression screening and referral protocols and existing breastfeeding education. Third, women in this sample were less depressed than other women in the database from which the sample was derived. Since postpartum depressive symptoms were predictive of breastfeeding in a less depressed group of women, an important next step would be to examine breastfeeding habits, and engagement with other home visitor promoted practices, in a more depressed group of women who might be less engaged in HV services.

\section{Conclusion}

In the present study, we sought to answer the question: Might MD interfere with mothers' ability to engage with a home visitor advised parenting practice-breastfeeding? This study produced several noteworthy findings that can inform prevention and intervention efforts aimed at optimizing the likelihood of breastfeeding for women enrolled in HV. First, women in this sample were less depressed than women enrolled in other HV programs. Second, rates of breastfeeding among women enrolled in DMHSP were higher than rates of breastfeeding reported in the general population. Third, Non-Hispanic White women were less likely to breastfeed than other racial groups. Fourth, the average EPDS score during the first 3 months postpartum predicted breastfeeding at 3 months postpartum. These findings highlight groups of women enrolled in HV for whom breastfeeding may be especially difficult. Mothers experiencing depressive symptoms in the early postpartum period may need additional support to increase their likelihood of breastfeeding. Further research is required to determine whether treatment of maternal depression is sufficient to increase the likelihood of breastfeeding for those who intend to.

Authorship statement - Francesca A. Scheiber: conceptualization, data curation, formal analysis, methodology, software, writing original draft, visualization; Lisa S. Segre: conceptualization, supervision, writing original draft, writing review and editing, visualization; Michael W. O'Hara: conceptualization, formal analysis, methodology, supervision, writing review and editing, visualization; Darby Taylor: resources, writing original draft; and Rebecca L. Brock: conceptualization, formal analysis, methodology, writing original draft, writing review and editing, visualization.

Declaration of competing interest - The authors have no conflicts of interest to report.

Acknowledgments - Francesca Scheiber was supported by the NIHGMS grant T32GM108540. The authors would like to thank the Des Moines Healthy Start and the Empowerment Family Support Project for their assistance during this project. 


\section{References}

American Academy of Pediatrics Section on Breastfeeding (2012). Breastfeeding and the use of human milk. Pediatrics, 129(3), e827-e841.

Ammerman, R. T., Putnam, F. W., Bosse, N. R., Teeters, A. R., \& Van Ginkel, J. B. (2010). Maternal depression in home visitation: A systematic review. Aggression and Violent Behavior, 15(3), 191-200.

Bergink, V., Kooistra, L., Lambregtse-van den Berg, M. P., Wijnen, H., Bunevicius, R., van Baar, A., \& Pop, V. (2011). Validation of the Edinburgh Depression Scale during pregnancy. Journal of Psychosomatic Research, 70(4), 385-389.

Binns, C., Lee, MiKyung, \& Low, W. Y. (2016). The Long-Term Public Health Benefits of Breastfeeding. Asia-Pacific Journal of Public Health, 28(1), 7-14.

Britton, C., McCormick, F. M., Renfrew, M. J., Wade, A., \& King, S. E. (2007). Support for breastfeeding mothers. Cochrane Database of Systematic Reviews(1).

Chung, M., Raman, G., Chew, P., Magula, N., Trikalinos, T., \& Lau, J. (2007). Breastfeeding and maternal and infant health outcomes in developed countries. Evidence Report/Technology Assessment (Full Rep), 153(153), 1-186.

Cicchetti, D., Rogosch, F. A., \& Toth, S. L. (2000). The efficacy of toddler-parent psychotherapy for fostering cognitive development in offspring of depressed mothers. Journal of Abnormal Child Psychology, 28(2), 135-148.

Cox, J. L., Holden, J. M., \& Sagovsky, R. (1987). Detection of Postnatal Depression: Development of the 10-item Edinburgh Postnatal Depression Scale. British Journal of Psychiatry, 150(6), 782-786.

Dennis, C.-L., \& McQueen, K. (2009). The Relationship Between Infant-Feeding Outcomes and Postpartum Depression: A Qualitative Systematic Review. Pediatrics, 123(4), e736-e751.

Dias, C. C., \& Figueiredo, B. (2015). Breastfeeding and depression: A systematic review of the literature. Journal of Affective Disorders, 171, 142-154.

Duffee, J. H., Mendelsohn, A. L., Kuo, A. A., Legano, L. A., \& Earls, M. F. (2017). Early Childhood Home Visiting. Pediatrics, 140(3), e20172150. https://doi.org/10.1542/peds.2017-2150.

Forman, D. R., O’Hara, M. W., Stuart, S., Gorman, L. L., Larsen, K. E., \& Coy, K. C. (2007). Effective treatment for postpartum depression is not sufficient to improve the developing mother-child relationship. Development Psychopathology, 19(02), https://doi.org/10.1017/S0954579407070289.

Forste, R., Weiss, J., \& Lippincott, E. (2001). The Decision to Breastfeed in the United States: Does Race Matter? Pediatrics, 108(2), 291-296.

Galler, J. R., Harrison, R. H., Ramsey, F., Chawla, S., \& Taylor, J. (2006). Postpartum feeding attitudes, maternal depression, and breastfeeding in Barbados. Infant Behavior and Development, 29(2), 189-203.

Gavin, N. I., Gaynes, B. N., Lohr, K. N., Meltzer-Brody, S., Gartlehner, G., \& Swinson, T. (2005). Perinatal Depression: A Systematic Review of Prevalence and Incidence. Obstetrics \& Gynecology, 106(5, Part 1), 1071-1083.

Gianni, M. L., Bettinelli, M. E., Manfra, P., Sorrentino, G., Bezze, E., Plevani, L., Cavallaro, G., Raffaeli, G., Crippa, B. L., \& Colombo, L. (2019). Breastfeeding difficulties and risk for early breastfeeding cessation. Nutrients, 11(10), 2266.

Goodman, J. H., Prager, J., Goldstein, R., \& Freeman, M. (2015). Perinatal Dyadic Psychotherapy for postpartum depression: A randomized controlled pilot trial. Archives Womens Mental Health, 18(3), 493-506.

Hatton, D. C., Harrison-Hohner, J., Coste, S., Dorato, V., Curet, L. B., \& McCarron, D. A. (2005). Symptoms of Postpartum Depression and Breastfeeding. Journal of Human Lactation, 21(4), 444-449. 
Healthy People. (2020). Maternal, Infant, and Child Health Objectives. Retrieved from https://www .healthypeople.gov/2020/topics-objectives/topic/maternal-infant-and-child-health/objectives.

Henderson, J. J., Evans, S. F., Straton, J. A. Y., Priest, S. R., \& Hagan, R. (2003). Impact of Postnatal Depression on Breastfeeding Duration. Birth Issues in Perinatal Care, 30(3), 175-180.

Holden, J. M., Sagovsky, R., \& Cox, J. L. (1989). Counselling in a general practice setting: Controlled study of health visitor intervention in treatment of postnatal depression. BMJ, 298(6668), 223-226.

Huang, Y.i., Ouyang, Y.-Q., \& Redding, S. R. (2019). Previous breastfeeding experience and its influence on breastfeeding outcomes in subsequent births: A systematic review. Women and Birth, 32(4), 303-309.

Hurley, K. M., Black, M. M., Papas, M. A., \& Quigg, A. M. (2008). Variation in breast-feeding behaviours, perceptions, and experiences by race/ethnicity among a low-income statewide sample of Special Supplemental Nutrition Program for Women, Infants, and Children (WIC) participants in the United States. Maternal and Child Nutrition, 4(2), 95-105.

Kitzman, H., Olds, D. L., Henderson, C. R., Hanks, C., Cole, R., Tatelbaum, R., .. . Barnard, K. (1997). Effect of Prenatal and Infancy Home Visitation by Nurses on Pregnancy Outcomes, Childhood Injuries, and Repeated Childbearing. JAMA, 278(8), 644-652.

Laisiriruangrai, P., Wiriyasirivaj, B., Phaloprakarn, C., \& Manusirivithaya, S. (2008). Prevalence of exclusive breastfeeding at 3, 4 and 6 months in Bangkok Metropolitan Administration Medical College and Vajira Hospital. Medical Journal of the Medical Association of Thailand, 91(7), 962.

Lau, C. Y. K., Lok, K. Y. W., \& Tarrant, M. (2018). Breastfeeding Duration and the Theory of Planned Behavior and Breastfeeding Self-Efficacy Framework: A Systematic Review of Observational Studies. Maternal and Child Health Journal, 22(3), 327-342.

Li, R., Darling, N., Maurice, E., Barker, L., \& Grummer-Strawn, L. M. (2005). Breastfeeding Rates in the United States by Characteristics of the Child, Mother, or Family: The 2002 National Immunization Survey. American Academy of Pediatrics.

Logsdon, M. C., Wisner, K., \& Hanusa, B. H. (2009). Does Maternal Role Functioning Improve with Antidepressant Treatment in Women with Postpartum Depression? Journal of Women's Health, 18(1), 85-90.

Love, J. M., Kisker, E. E., Ross, C., Raikes, H., Constantine, J., Boller, K., . . Brady-Smith, C. (2005). The effectiveness of early head start for 3-year-old children and their parents: lessons for policy and programs. Developmental psychology, 41(6), 885.

McBride, H. L., Wiens, R. M., McDonald, M. J., Cox, D. W., \& Chan, E. K. (2014). The Edinburgh Postnatal Depression Scale (EPDS): A review of the reported validity evidence. Validity and validation in social, behavioral, and health sciences (pp. 157-174). Springer.

Mejdoubi, J., van den Heijkant, S. C. C. M., van Leerdam, F. J. M., Crone, M., Crijnen, A., \& HiraSing, R. A. (2014). Effects of nurse home visitation on cigarette smoking, pregnancy outcomes and breastfeeding: A randomized controlled trial. Midwifery, 30(6), 688-695.

National Home Visiting Resource Center. (2018). Home Visiting Yearbook. In James Bell Associates \& Urban Institute (Eds.). Arlington, Virginia.

Ogbuanu, C., Glover, S., Probst, J., Liu, J., \& Hussey, J. (2011). The Effect of Maternity Leave Length and Time of Return to Work on Breastfeeding. Pediatrics, 127(6), e1414-e1427.

O'Hara, M. W., \& Swain, A. M. (1996). Rates and risk of postpartum depression-a meta-analysis. International Review of Psychiatry, 8(1), 37-54.

Olds, D. L., Robinson, J., O'Brien, R., Luckey, D. W., Pettitt, L. M., Henderson, C. R., Ng, R. K., Sheff, K. L., Korfmacher, J., Hiatt, S., \& Talmi, A. (2002). Home Visiting by Paraprofessionals and by Nurses: A Randomized, Controlled Trial. Pediatrics, 110(3), 486-496. 
Raj, V. K., \& Plichta, S. B. (1998). The Role of Social Support in Breastfeeding Promotion: A Literature Review. Journal of Human Lactation, 14(1), 41-45.

Segre, L. S., Brock, R. L., \& O'Hara, M. W. (2015). Depression treatment for impoverished mothers by point-of-care providers: A randomized controlled trial. Journal of Consulting and Clinical Psychology, 83(2), 314-324. https://doi.org/10.1037/a0038495.

Segre, L. S., O'Hara, M. W., Brock, R. L., \& Taylor, D. (2012). Depression Screening of Perinatal Women by the Des Moines Healthy Start Project: Program Description and Evaluation. PS, 63(3), 250-255.

Segre, L. S., Stasik, S. M., O'Hara, M. W., \& Arndt, S. (2010). Listening Visits: An evaluation of the effectiveness and acceptability of a home-based depression treatment. Psychotherapy Research, 20(6), 712-721.

Segre, L. S., \& Taylor, D. (2014). Implementing Universal Maternal Depression Screening in Home Visiting Programs: A Pragmatic Overview. Zero to Three, 34(5), 12-19.

Shah, M. K., \& Austin, K. R. (2014). Do Home Visiting Services Received During Pregnancy Improve Birth Outcomes? Findings from Virginia PRAMS 2007-2008. Public Health Nursing, 31(5), 405-413.

Slomian, J., Honvo, G., Emonts, P., Reginster, J.-Y., \& Bruyere, O. (2019). Consequences of maternal postpartum depression: A systematic review of maternal and infant outcomes. Women's Health, 15, 1-55.

Stevens, J., Ammerman, R. T., Putnam, F. G., \& Van Ginkel, J. B. (2002). Depression and trauma history in first-time mothers receiving home visitation. Journal of Community Psychology, 30(5), 551-564.

Stuebe, A. M., Rich-Edwards, J. W., Willett, W. C., Manson, J. E., \& Michels, K. B. (2005). Duration of lactation and incidence of type 2 diabetes. JAMA, 294(20), 2601-2610.

Victora, C. G., Bahl, R., Barros, A. J. D., Franca, G. V. A., Horton, S., Krasevec, J., . . Rollins, N. C. (2016). Breastfeeeding in the 21st century: Epidemiology, mechanisms, and lifelong effect. The Lancet, 387, 475-490.

Watkins, S., Meltzer-Brody, S., Zolnoun, D., \& Stuebe, A. (2011). Early Breastfeeding Experiences and Postpartum Depression. Obstetrics \& Gynecology, 118(2), 214-221.

World Health Organization. (2011). Exclusive breastfeeding for six months best for babies from https://www.who.int/mediacentre/news/statements/2011/breastfeeding_20110115/en/. 